\title{
Alterações congênitas do coração e dos grandes vasos em cães ${ }^{1}$
}

\author{
Fernando F. Argenta ${ }^{2}$, Saulo P. Pavarini ${ }^{2}$, David Driemeier ${ }^{2}$ e Luciana Sonne $^{2 *}$
}

\begin{abstract}
Argenta F.F., Pavarini S.P., Driemeier D. \& Sonne L. 2018. [Congenital abnormalities of the heart and large vessels of dogs]. Alterações congênitas do coração e dos grandes vasos em cães. Pesquisa Veterinária Brasileira 38(6)1184-1189. Setor de Patologia Veterinária, Departamento de Patologia Clínica Veterinária, Faculdade de Veterinária, Universidade Federal do Rio Grande do Sul, Av. Bento Gonçalves 9090, prédio 42505, Porto Alegre, RS 91540-000, Brazil. E-mail: lusonne@yahoo.com.br

Congenital alterations of the heart and large vessels (CAHLV) are defined as morphological defects associated with birth and are the most frequent congenital anomalies of domestic animals. The aim of this study was to determine the frequency of CAHLV in dogs in Rio Grande do Sul, with emphasis in the Metropolitan Region of Porto Alegre, from January 2000 to December 2016. Of the 7,903 necropsied dogs, 27 died spontaneously or were submitted to euthanasia due to CAHLV, representing $0.3 \%$ of the cases. In $11.1 \%$ of the dogs, there were two congenital disorders in the heart or in the great vessels, totaling 30 changes. The age of affected dogs ranged from one day to 12 years, with the median age of four months. In $81.5 \%$ it affected dogs with breed, and $18.5 \%$, without breed defined. In $51.8 \%$ were males, and $48.2 \%$, females. Subaortic stenosis was the most frequent alteration, followed by atrial septal defect, persistent right aortic arch, patent ductus arteriosus, pulmonic stenosis, ventricular septal defect, and endocardial fibroelastosis. Of the multiple cases, the combinations found were: patent ductus arteriosus associated with atrial septal defect, subaortic stenosis with ventricular septal defect, and atrial and ventricular septal defect.
\end{abstract}

INDEX TERMS: Heart disorders, congenital disorders, subaortic and pulmonic stenosis, atrial and septal defect, persistent right aortic arch, patent ductus arteriosus, dogs.

RESUMO.- As alterações congênitas do coração e dos grandes vasos (ACCGV) são definidas como defeitos morfológicos associados ao nascimento e são as anomalias congênitas mais frequentes dos animais domésticos. 0 objetivo deste trabalho foi determinar a frequência de diagnósticos de ACCGV em cães no Rio Grande do Sul, com ênfase na Região Metropolitana de Porto Alegre, no período de janeiro de 2000 a dezembro de 2016. Do total de 7.903 necropsias de cães, 27 morreram espontaneamente ou foram submetidos à eutanásia devido às ACCGV, representando $0,3 \%$ dos casos. Em $11,1 \%$ dos cães apresentaram dois distúrbios congênitos no coração ou nos grandes vasos, totalizando 30 alterações. A idade variou de um dia a 12 anos, com a idade mediana de quatro meses. Em 81,5\% acometeu cães com raça, e 18,5\% sem raça

\footnotetext{
${ }^{1}$ Recebido em 26 de maio de 2017.

Aceito para publicação em 14 de junho de 2017.

${ }^{2}$ Setor de Patologia Veterinária, Faculdade de Veterinária, Universidade Federal do Rio Grande do Sul (UFRGS), Av. Bento Gonçalves 9090, prédio 42505, Porto Alegre, RS 91540-000, Brasil. *Autor para correspondência: lusonne@yahoo.com.br
}

definida. Em relação ao sexo, 51,8\% dos cães eram machos e 48,2\%, fêmeas. Estenose subaórtica foi a alteração mais frequente, seguido por defeito do septo atrial, persistência do arco aórtico direito, persistência do ducto arterioso, estenose pulmonar e defeito do septo interventricular, e fibroelastose endocárdica. Dos casos múltiplos, as combinações encontradas foram: persistência do ducto arterioso associado com defeito do septo atrial, estenose subaórtica com defeito do septo interventricular, e defeito do septo atrial e ventricular.

TERMOS DE INDEXAÇÃO: Coração, distúrbios cardíacos congênitos, estenose subaórtica e pulmonar, defeito de septo, persistência do arco aórtico, persistência ducto arterioso, caninos.

\section{INTRODUÇÃO}

As alterações congênitas do coração e dos grandes vasos (ACCGV) são definidas como defeitos morfológicos associados ao nascimento (MacDonald 2006) e são as anomalias congênitas mais frequentes dos animais domésticos (Ocarino et al. 2016). Diversos processos estão envolvidos na patogênese dessas 
alterações, como etiologias tóxicas, físicas ou nutricionais (Miller et al. 2013, Ocarino et al. 2016), porém em cães, são frequentemente hereditárias (Robinson \& Robinson 2016). A gravidade dos sinais clínicos depende do grau da lesão, muitas vezes podem levar ao surgimento rápido de sinais clínicos de insuficiência cardíaca ou podem possibilitar que o cão chegue até a idade adulta (Ocarino et al. 2016). As ACCGV podem ser derivadas de falhas no fechamento das comunicações cardiovasculares fetais, como a persistência do ducto arterioso e defeitos nos septos atrial e interventricular; falhas no desenvolvimento valvular normal, como a estenose pulmonar e subaórtica; mau posicionamento de grandes vasos, como a persistência do arco aórtico direito, e outras anomalias, como a fibroelastose do endocárdio (Miller et al. 2013). 0 reconhecimento precoce das ACCGV é de grande importância, pois fornece um prognóstico favorável, visto que algumas anomalias podem ser corrigidas cirurgicamente (Darke 1989, Oliveira et al. 2011).

Este trabalho tem como objetivo determinar a frequência das ACCGV em cães no Rio Grande do Sul, com ênfase na Região Metropolitana de Porto Alegre, no período de 16 anos, abordando seus aspectos epidemiológicos e patológicos.

\section{MATERIAL E MÉTODOS}

Foram revisados os laudos de necropsias de caninos arquivados do Setor de Patologia Veterinária da Universidade Federal do Rio Grande do Sul (SPV-UFRGS) no período de janeiro de 2000 a dezembro de 2016, e selecionados para o estudo os casos diagnosticados com alterações congênitas do coração e dos grandes vasos. Dados referentes à identificação do cão, como raça, sexo e idade, além do histórico clínico, lesões macroscópicas e microscópicas, e os diagnósticos foram analisados e compilados.

\section{RESULTADOS}

Foram analisados laudos de necropsia de 7.903 cães, destes, 685 morreram ou foram submetidos à eutanásia devido a doenças cardiovasculares, dentre estas, as alterações congênitas foram diagnosticadas em 3,9\% (27/685) dos casos, e em relação ao número total de necropsias a frequência foi de 0,3\% (27/7903). Dos 27 cães, 11,1\% (3/27) apresentaram combinação de dois distúrbios congênitos no coração ou nos grandes vasos, totalizando 30 alterações. Do total dos casos analisados, 96,5\% (26/27) eram oriundos da Região Metropolitana de Porto Alegre, e nos 3,7\% (1/27) restantes, Região Nordeste Rio-grandense.

De uma maneira geral, a idade variou de um dia a 12 anos, com a mediana de quatro meses (idade média de 22,2 meses). Em relação ao sexo, 51,8\% (14/27) eram machos e 48,2\% (13/27) fêmeas. Em 81,5\% (22/27) dos casos acometeram cães com raça, e os 18,5\% (5/27) restantes, sem raça definida (SRD). Dentre as raças, Boxer, Buldogue francês, Dachshund, Fila brasileiro e Pinscher foram as mais acometidas, representando 9,1\% (2/22) dos casos. 0 diagnóstico, número de casos e a porcentagem das ACCGV em cães estão detalhados no Quadro 1.

\section{Estenose subaórtica}

Do total de 30 ACCGV, 11 cães foram diagnosticados com estenose subaórtica, representando 36,7\% dos casos. Quanto ao sexo, 63,6\% (7/11) eram fêmeas e 36,4\% (4/11), machos. A idade variou de dois meses a 12 anos, com a idade mediana de quatro meses (média de 37,2 meses). Em 81,8\% (9/11) dos casos afetou cães com raça, e os 18,2\% (2/11) restantes, SRD. As raças acometidas foram Dachshund (18,2\% (2/11), e Dálmata, Yorkshire terrier, Pinscher, Boxer, Lhasa Apso, Rotweiller e Sharpei (9,1\% (1/11), cada). Dos casos combinados, em um cão observou persistência do forme oval concomitante com a estenose subaórtica.

Em 54,5\% (6/11) dos casos diagnosticados com estenose subaórtica foi relatada morte súbita. Em relação aos sinais clínicos descritos pelos tutores/veterinários clínicos, 45,5\% (5/11) dos cães apresentaram dificuldade respiratória, e cianose e secreção nasal, em 9,1\% (1/11), cada.

À necropsia, todos os casos apresentaram uma camada espessa de tecido conjuntivo fibroso no ventrículo esquerdo abaixo das válvulas semilunares aórticas (Fig.1A), com consequente hipertrofia do ventrículo esquerdo e discreta dilatação da aorta. Em relação às lesões extracardíacas, todos os cães apresentaram graus variados de edema e congestão pulmonares, com inúmeros macrófagos alveolares contendo pigmento acastanhado (hemossiderina) em seus interiores (células da falha cardíaca) identificados à microscopia. Congestão passiva crônica do fígado e ascite foram relatadas em $63,6 \%$ (7/11) e 18,2\% (2/11), respectivamente.

\section{Defeito do septo atrial}

Sete cães foram diagnosticados com defeito do septo atrial, representando 23,3\% do total das ACCGV. Destes, 71,4\% (5/7) eram machos e 28,6\% (2/7), fêmeas. A idade variou de um dia a três anos, com a idade mediana de cinco meses (média de 8,8 meses). Todos os cães eram de raça definida, sendo estas: Buldogue inglês, Buldogue francês, Spitz alemão, Fila brasileiro, Bichon Frisé, Pitbull e Boxer, representando 14,3\% (1/7) cada. Em 28,6\% (2/7) dos casos identificaram alterações congênitas combinadas, como persistência do ducto arterioso $(14,3 \%, 1 / 7)$ e defeito interventricular $(14,3 \%, 1 / 7)$ concomitantes com o defeito do septo atrial.

Morte súbita foi relatada em 42,8\% (3/7) dos casos e os principais sinais clínicos relatados foram dificuldade respiratória, descrita em 42,3\% (3/7) dos casos, seguido por secreção nasal e cianose, com 28,6\% (2/7) e 14,3\% (1/7), respectivamente.

À necropsia identificou-se nos sete casos, um orifício de diâmetro variado comunicando os dois átrios (Fig.1B) com consequente dilatação do ventrículo direito. Em relação às

Quadro 1. Alterações congênitas do coração e dos grandes vasos, número de casos e porcentagem de cães diagnosticados no Setor de Patologia Veterinária, UFRGS

\begin{tabular}{lcc}
\hline \multicolumn{1}{c}{ Diagnóstico } & $\begin{array}{c}\text { Número de } \\
\text { casos }\end{array}$ & $\begin{array}{c}\text { Porcentagem } \\
(\%)\end{array}$ \\
\hline Estenose subaórtica & 11 & 36,7 \\
Defeito do septo atrial & 7 & 23,3 \\
Persistência do arco aórtico direito & 4 & 13,3 \\
Persistência do ducto arterioso & 3 & 10,0 \\
Estenose pulmonar & 2 & 6,7 \\
Defeito do septo interventricular & 2 & 6,7 \\
Fibroelastose endocárdica & 1 & 3,3 \\
TOTAL & 30 & 100
\end{tabular}




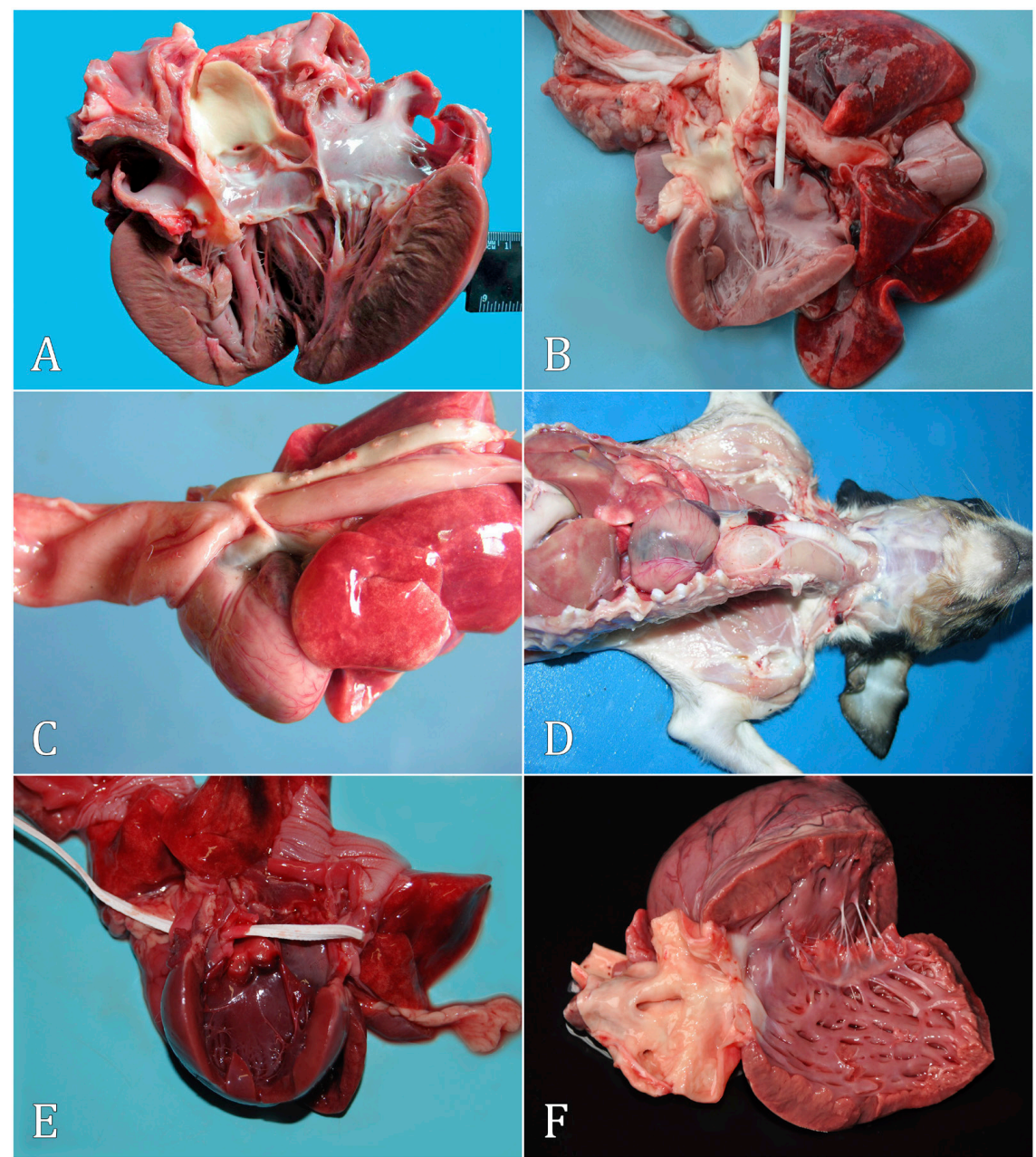

Fig.1. Aspectos macroscópicos das alterações congênitas do coração e de grandes vasos em cães. (A) Estenose subaórtica com camada de tecido conjuntivo fibroso esbranquiçado no ventrículo esquerdo abaixo das válvulas semilunares aórticas. (B) Defeito do septo atrial, caracterizado por orifício comunicando os dois átrios. (C) Persistência do arco aórtico direito, caracterizada pela formação de um ligamento fibroso sobre o esôfago e traqueia, causando a dilatação da porção cranial esofágica. (D) Megaesôfago no cão com persistência do arco aórtico direito da figura anterior. (E) Persistência do ducto arterioso, comunicação à aorta e à artéria pulmonar (F) Estenose pulmonar com camada espessa de tecido conjuntivo fibroso abaixo das válvulas semilunares pulmonares, com dilatação da artéria pulmonar.

lesões extracardíacas, 85,7\% (6/7) dos casos apresentaram graus variados de edema e congestão pulmonares, com inúmeras células da falha cardíaca no interior de alvéolos, identificadas à microscopia. Congestão passiva crônica do fígado, ascite e hidrotórax foram identificadas em $42,3 \%$ (3/7), 14,3\% (1/7) e 14,3\% (1/7), respectivamente.

\section{Persistência do arco aórtico direito}

Quatro cães foram diagnosticados com persistência do arco aórtico direito, representando 13,3\%. Essa anomalia afetou $75 \%$ (3/4) fêmeas, e 25\% (1/7), machos. A idade dos cães acometidos variou de dois meses a seis meses, com a idade mediana de três meses (média de 3,5 meses). Quanto à raça, $50 \%(2 / 4)$ eram SRD, e os outros $50 \%$, com raça, sendo essas, Buldogue francês e Pinscher, representando 25\% (1/4) cada.

Os sinais clínicos relatados nestes casos foram caracterizados por regurgitação, identificada em 100\% (4/4) dos cães acometidos, seguido por cianose e dificuldade respiratória, descrito em $25 \%$ (1/4), cada.

À necropsia, nos quatro casos observou-se a persistência do arco aórtico direito, caracterizada pela formação de um ligamento fibroso sobre o esôfago e traqueia (Fig.1C), causando a dilatação da porção cranial esofágica (megaesôfago) (Fig.1D). Em 50\% (2/4) dos cães apresentaram áreas multifocais de consolidação pulmonar, que à microscopia, caracterizaram-se por broncopneumonia por aspiração.

\section{Persistência do ducto arterioso}

Três cães apresentaram persistência do ducto arterioso (10\%), destes, 66,7\% (2/3) eram fêmeas e 33,3\% (1/3) machos. A idade variou de 10 dias a quatro meses, com a mediana de três meses (média de 2,4 meses). Todos os cães apresentavam raça definida, sendo estas: Buldogue francês, Maltês e Shih Tzu, representando 33,3\% (1/3) cada. Em 33,3\% (1/3) dos 
casos observou persistência do ducto arterioso concomitante com defeito do septo atrial.

Morte súbita foi relatada em 33,3\% (1/3), e dificuldade respiratória foi o sinal clínico identificado em 66,7\% (2/3).

Observou-se na necropsia dos três casos, comunicação entre a aorta e a artéria pulmonar sem a formação do ligamentum arteriosum (Fig.1E), com dilatação destas e consequente hipertrofia das câmaras cardíacas esquerda e direita. Edema e congestão pulmonares, além de células da falha cardíaca, foram relatados em todos os casos, e congestão passiva crônica do fígado em 33,3\% (1/3).

\section{Estenose pulmonar}

Dois cães foram diagnosticados com estenose pulmonar $(6,7 \%)$, sendo estes, machos, de raça definida (Poodle e Fila brasileiro). Em um caso a idade foi de seis meses, e no outro, oito anos. Não foram relatadas informações referentes aos sinais clínicos.

Macroscopicamente, os dois cães apresentaram estreitamento do lúmen da artéria pulmonar, devido a camada espessa de tecido conjuntivo fibroso abaixo das válvulas semilunares pulmonares, com consequente hipertrofia concêntrica do ventrículo direito e dilatação da artéria pulmonar (Fig.1F). Em um caso se observou acentuado edema e congestão pulmonares, com células da falha cardíaca, ascite e congestão passiva crônica do fígado.

\section{Defeito do septo interventricular}

Dois cães foram diagnosticados com defeito do septo interventricular (6,7\%). Um caso acometeu fêmea SRD de dois meses de idade, e o outro, macho da raça Bichon Frisé com três meses de idade. Os dois cães apresentavam combinações das ACCGV, sendo estas: estenose subaórtica, e defeito do septo atrial concomitantes com o defeito do septo interventricular.

Morte súbita foi relatada na fêmea. Os sinais clínicos foram descritos no cão macho e foram caracterizados por dificuldade respiratória, secreção nasal e cianose.

Na necropsia observou um orifício de tamanho variado no septo interventricular, envolvendo a porção membranosa, com hipertrofia excêntrica do ventrículo esquerdo e hipertrofia concêntrica do ventrículo direito. Os dois cães apresentaram graus variados de edema e congestão pulmonares, com células da falha cardíaca identificadas à microscopia.

\section{Fibroelastose endocárdica}

Um cão foi diagnosticado com fibroelastose endocárdica, representando 3,3\% do total das ACCGV. 0 cão era macho, SRD e de dois meses de idade. Dificuldade respiratória, secreção nasal e cianose foram os sinais clínicos relatados.

Macroscopicamente, o coração do canino afetado apresentou múltiplas e moderadas áreas esbranquiçadas no endocárdio, com hipertrofia secundária dos ventrículos e dilatação do átrio direito. Ascite, hidrotórax, edema e congestão pulmonares e congestão passiva crônica do fígado foram as lesões extracardíacas identificadas.

\section{DISCUSSÃO}

No presente trabalho, a frequência das ACCGV diagnosticadas através da necropsia foi de $0,3 \%$ do total de necropsias de cães, e em relação às doenças cardiovasculares, representou $3,9 \%$ dos casos. Dado semelhante é descrito na literatura, onde a frequência foi de aproximadamente $0,5 \%$, porém, os casos foram analisados através do exame post mortem, e também pela avaliação clínica cardíaca (Patterson 1968, Mulvihill \& Priester 1973). Em diversos estudos retrospectivos realizados através da análise clínica de cães com doenças cardiovasculares, a frequência de ACCGV variou de $17 \%$ a 23,5\% (Baumgartner \& Glaus 2003, Oliveira et al. 2011). Na pesquisa de Oliveira et al. (2011), apenas 4,4\% dos cães com ACCGV foram diagnosticados através da necropsia.

A combinação entre duas ACCGV foi identificada em $11,1 \%$ dos casos. Em pesquisas semelhantes, a frequência de casos combinados variou de 8,2\% a 15\% (Baumgartner \& Glaus 2003, Oliveira et al. 2011). Os casos de defeito do septo interventricular do presente estudo estavam associados com outras ACCGV. Robinson \& Robinson (2016) descrevem que esse defeito, embora ocorra mais comumente como uma alteração isolada, em muitas ocasiões observa-se combinação, como ocorre na tetralogia de Fallot ou na persistência do ducto arterioso.

A idade dos caninos com ACCGV variou de um dia a 12 anos, com a mediana de quatro meses (idade média de 22,2 meses). Em pesquisas semelhantes, a idade média variou de 11 a 42 meses (Tidholm 1997, Oliveira et al. 2011). 0 diagnóstico de ACCGV em cães ocorre frequentemente antes do primeiro ano de vida (Mulvihill \& Priester 1973, Buchanan 1999), porém, cães com ACCGV podem atingir a fase adulta, pois a gravidade dos sinais clínicos está relacionada com o grau da lesão (Ocarino et al. 2016).

Em relação à predisposição sexual, pesquisadores descreveram que a estenose pulmonar é mais comum em machos (Tidholm 1997, Oliveira et al. 2011) e a persistência do ducto arterioso, em fêmeas (Tidholm 1997, Oliveira et al. 2011, Miller et al. 2013), dados que corroboram com o presente trabalho.

Cães com raça foram os mais acometidos, semelhante ao descrito por Oliveira et al. (2011), e isto provavelmente ocorre por questões de consanguinidade das raças. No presente estudo, Boxer, Buldogue francês, Dachshund, Fila brasileiro e Pinscher foram as mais acometidas. Pesquisadores sugerem que a estenose subaórtica é mais comum em raças de grande porte, como Pastor alemão, Golden retriever e Rottweiler (MacDonald 2006, Miller et al. 2013, Robinson \& Robinson 2016). Boxer é uma das principais raças predispostas ao desenvolvimento de ACCGV (Chetboul et al. 2006, Bussadori et al. 2009, Oliveira et al. 2011), e Pinscher e Buldogue francês foram frequentemente diagnosticados com ACCGV em um estudo semelhante (Oliveira et al. 2011).

Estenose subaórtica foi a alteração mais frequente, este dado está de acordo com os descritos na literatura (Tidholm 1997, Baumgartner \& Glaus 2003, Egenvall et al. 2006, MacDonald 2006). As falhas no desenvolvimento valvular, como a estenose subaórtica e estenose pulmonar, juntamente com a persistência do ducto arterioso, são as ACCGV mais comuns em cães, conforme relatado por diversos pesquisadores (Patterson 1968, Buchanan 1999, Oliveira et al. 2011, Miller et al. 2013, Robinson \& Robinson 2016). No presente estudo, essas alterações representaram mais de 50\% dos diagnósticos.

Dificuldade respiratória, secreção nasal e cianose foram os principais sinais clínicos descritos. Regurgitação foi relatada nos cães com persistência do arco aórtico direito no presente estudo. A maioria dos casos de ACCGV é assintomática, porém, 
sinais relacionados com insuficiência cardiorrespiratória, como dispneia (Tidholm 1997, Miller et al. 2013), síncope, intolerância ao exercício, cianose, taquipneia e tosse podem ser observados (MacDonald 2006). A gravidade dos sinais clínicos é variável e depende do grau da lesão (Ocarino et al. 2016). Em alguns casos, o defeito pode ser incompatível com a vida (Miller etal. 2013). Diversos pesquisadores relatam que morte súbita pode ocorrer nas ACCGV, principalmente, na estenose subaórtica e pulmonar (Kienle et al. 1994, MacDonald 2006, Robinson \& Robinson 2016). No presente estudo, morte súbita foi relatada pelos tutores/veterinário clínico em 11 cães, representando 40,7\%.

Macroscopicamente, todas as ACCGV encontradas no presente estudo corroboram com os achados descritos na literatura (Miller et al. 2013, Ocarino et al. 2016, Robinson \& Robinson 2016). Hipertrofia do miocárdio e dilatação vascular sobrejacente à estenose foram achados frequentes. Para Robinson \& Robinson (2016), a maioria dos casos de ACCGV reflete-se no aumento grosseiro do coração e na alteração do tamanho ou disposição dos grandes vasos. A hipertrofia concêntrica nos cães com estenose subaórtica e pulmonar do presente estudo está relacionada com a sobrecarga de pressão decorrente da obstrução do fluxo sanguíneo (Ocarino et al. 2016), e nos casos de defeitos de septos, os ventrículos sofreram hipertrofia excêntrica devido à sobrecarga de volume, e concêntrica por causa da sobrecarga de pressão (Robinson \& Robinson 2016). No presente estudo, todos os cães com defeito no septo interventricular apresentavam um orifício na porção superior (membranosa), que é o local mais frequente deste achado (Miller et al. 2013). Nos casos de persistência do ducto arterioso, a hipertrofia do ventrículo esquerdo e direito ocorreu em razão do desvio de sangue do lado esquerdo para o direito, com sobrecarga do volume sanguíneo e aumento de pressão (Ocarino et al. 2016). 0 megaesôfago identificado em todos os casos de persistência do arco aórtico direito é causado pela compressão do ligamento sobre o esôfago, comprimindo-o sobre a traqueia, no qual ocasiona dilatação esofágica e regurgitação (Ocarino et al. 2016). Na fibroelastose endocárdica, há múltiplas áreas esbranquiçadas no endocárdio, que microscopicamente, consistem em deposição de tecido conjuntivo fibroso (Larsson et al. 1997), assim como relatado no caso do presente estudo.

Edema e congestão pulmonares, ascite, e congestão passiva crônica do fígado foram lesões extracardíacas frequentes no presente estudo, e são comuns em casos de insuficiência cardíaca em cães (Ocarino et al. 2016, Robinson \& Robinson 2016). A formação de edema nestes casos ocorreu principalmente pelo aumento da pressão hidrostática, decorrente da estase do fluxo sanguíneo (Ocarino et al. 2016). Células da falha cardíaca nos alvéolos pulmonares, identificadas à microscopia, foi um achado comum nos cães com ACCGV. Estas células são originadas devido ao aumento na eritrofagocitose, decorrente da estase do fluxo sanguíneo no leito capilar pulmonar nos casos de insuficiência cardíaca. As lesões hepáticas identificadas também estão relacionadas com a hipertensão portal consequente da insuficiência cardíaca (Miller et al. 2013). Dois cães com persistência do arco aórtico direito apresentaram concomitantemente, broncopneumonia por aspiração, e isso pode estar relacionado com a dificuldade de deglutição nestes animais, decorrente a dilatação esofágica presente nessa alteração (Miller et al. 2013).

\section{CONCLUSÕES}

As alterações congênitas do coração e dos grandes vasos (ACCGV) são doenças importantes em cães de raças definidas. A idade é bastante variável, com a idade mediana de quatro meses. Clinicamente caracterizam-se por sinais de insuficiência cardíaca, como dificuldade respiratória, secreção nasal e cianose, e morte súbita.

Estenose subaórtica foi o defeito mais comumente encontrado, seguido por defeito do septo atrial e persistência do arco aórtico direito. Múltiplos defeitos podem ocorrer no mesmo cão.

As combinações encontradas frequentemente são: persistência do ducto arterioso associada com o defeito do septo atrial, estenose subaórtica com defeito do septo interventricular, e defeito atrial e ventricular.

Edema e congestão pulmonares, ascite, e congestão passiva crônica do fígado são lesões extracardíacas frequentes nos casos de ACCGV.

O conhecimento da frequência destas anomalias é fundamental para o diagnóstico diferencial e para determinar um tratamento adequado.

\section{REFERÊNCIAS}

Baumgartner C. \& Glaus T.M. 2003. Angeborene herzerkrankungen beim hund: eine retrospektive analyse. Schweiz. Arch. Tierheilkunde 145(11):527-536. http://dx.doi.org/10.1024/0036-7281.145.11.527.

Buchanan J.W. 1999. Prevalence of cardiovascular disorders, p.458-463. In: Fox P.R., Sisson D.D. \& Moise N.S. (Eds), Textbook of Canine and Feline Cardiology. 2nd ed. W.B. Saunders, Philadelphia.

Bussadori C., Pradelli D., Borgarelli M., Chiavegato D., D’Agnolo G., Menegazzo L., Migliorini F., Santilli R., Zani A. \& Quintavalla C. 2009. Congenital heart disease in boxer dogs: results of 6 years of breed screening. Vet. J. 181(2):187192. http://dx.doi.org/10.1016/j.tvjl.2008.02.008. PMid:18400530.

Chetboul V., Trollé J.M., Nicolle A., Carlos Sampedrano C., Gouni V., Laforge H., Benalloul T., Tissier R. \& Pouchelon J.L. 2006. Congenital heart diseases in the Boxer dog: a retrospective study of 105 cases (1998-2005). J. Vet. Med. Physiol. Pathol. Clin. Med.53(7):346-351. http://dx.doi.org/10.1111/j.14390442.2006.00865.x. PMid:16922831.

Darke P.G.G. 1989. Congenital heart disease in dogs and cats. J. Small Anim. Pract. 30(1):599-607. http://dx.doi.org/10.1111/j.1748-5827.1989. tb01485.x

Egenvall A., Bonnett B.N. \& Häggström J. 2006. Heart disease as a cause of death in insured Swedish dogs younger than 10 years of age. J. Vet. Intern. Med. 20(4):894-903. http://dx.doi.org/10.1111/j.1939-1676.2006. tb01803.x. PMid:16955814.

Kienle R.D., Thomas W.P. \& Pion P.D. 1994. The natural history of canine congenital subaortic stenosis. J. Vet. Intern. Med. 8(6):423-431. http:// dx.doi.org/10.1111/j.1939-1676.1994.tb03262.x. PMid:7884729.

Larsson M.H., Baccaro M.R., Pereira L. \& Oliveira S.M. 1997. Endocardial fibroelastosis in a dog. J. Small Anim. Pract. 38(4):168-170. http://dx.doi. org/10.1111/j.1748-5827.1997.tb03456.x. PMid:9127286.

MacDonald K.A. 2006. Congenital heart diseases of puppies and kittens. Vet. Clin. N. Am. Small Anim. Pract. 36(3):503-531, vi. http://dx.doi. org/10.1016/j.cvsm.2005.12.006. PMid:16564411.

Miller M., Van Vleet J.F. \& Gal A. 2013. Sistema cardiovascular, p.559-614. In: McGavin M.D. \& Zachary J.F. (Eds), Bases da Patologia em Veterinária. 5a ed. Elsevier, Rio de Janeiro. 
Mulvihill J.J. \& Priester W.A. 1973. Congenital heart disease in dogs: epidemiologic similarities to man. Teratology 7(1):73-78. http://dx.doi. org/10.1002/tera.1420070111. PMid:4693746.

Ocarino N.M., Paixão T.A., Carvalho E.C.Q. \& Gimeno E.J. 2016. Sistema Cardiovascular, Cap2. p.49-85. In: Santos R.L. \& Alessi A.C. (Eds), Patologia Veterinária. $2^{\underline{a}}$ ed. Roca, Rio de Janeiro.

Oliveira P., Domenech O., Silva J., Vannini S., Bussadori R. \& Bussadori C. 2011. Retrospective review of congenital heart disease in 976 dogs. J. Vet. Intern. Med. 25(3):477-483. http://dx.doi.org/10.1111/j.1939-1676.2011.0711.x. PMid:21418326.
Patterson D.F. 1968. Epidemiologic and genetic studies of congenital heart disease in the dog. Circ. Res. 23(2):171-202. http://dx.doi.org/10.1161/01. RES.23.2.171. PMid:5662573.

Robinson W.F. \& Robinson N.A. 2016. Cardiovascular system, p.14-24. In: Maxie M.G. (Ed.), Jubb, Kennedy and Palmer's Pathology of Domestic Animals. Vol.3. 6th ed. Elsevier, St Louis.

Tidholm A. 1997. Retrospective study of congenital heart defects in 151 dogs. J. Small Anim. Pract. 38(3):94-98. http://dx.doi.org/10.1111/j.1748-5827.1997. tb03326.x. PMid:9097239. 